\title{
Vaccines against intracellular infections requiring cellular immunity
}

\author{
Robert A. Seder ${ }^{\star}$ Adrian V. S. Hill ${ }^{\dagger}$ \\ ${ }^{\star}$ Clinical Immunology Section, Laboratory of Clinical Investigation, National Institute of Allergy and Infectious Diseases, National Institutes of \\ Health, Bethesda, Maryland 20892, USA \\ $\dagger$ Molecular Immunology Group, Institute of Molecular Medicine, Nuffield Department of Medicine, University of Oxford, John Radcliffe Hospital, \\ Oxford OX3 9DU, UK
}

Vaccines against a variety of infectious diseases represent one of the great triumphs of medicine. The immune correlates of protection induced by most current vaccines seem to be mediated by long-lived humoral immune responses. By contrast, there are no currently available vaccines that are uniformly effective for diseases such as HIV, malaria and tuberculosis, in which the cellular immune response might be crucial in mediating protection. Here we examine the mechanisms by which long-lived cellular immune responses are generated and maintained in vivo. We then discuss current approaches for vaccination against diseases in which cellular immune responses are important for protection.

he origins of acquired immunity against
infectious disease were observed more than
2,000 years ago. In $430 \mathrm{BC}$, Thucydides described
the plague of Athens in his historical account of
the Peloponnesian war:

It was with those who had recovered from the disease that the sick and the dying found most compassion. These knew what it was from experience, and had now no fear for themselves; for the same man was never attacked twice-never at least fatally.

At the end of the eighteenth century, Jenner's observation that vaccination with cowpox virus protected individuals from smallpox provided the scientific basis for the concept of vaccination. Today, vaccines for a variety of bacterial and viral diseases have had a major impact worldwide in reducing both morbidity and mortality. The main reason for the success of current vaccines is that, for most viral and bacterial infections, primary protection is thought to be mediated by a long-lived humoral immune response through the production of antibodies. It is noteworthy that such responses are readily induced by many different vaccine formulations. By contrast, for infections such as tuberculosis, malaria and HIV, which now bring about a substantial proportion of the deaths worldwide from infection, there are no uniformly effective vaccines. Furthermore, although humoral immunity could be important in preventing infection by HIV and certain stages of malaria infection, it is the cellular immune response that is central to the mediation of protection in all of these diseases. The focus of this review is our understanding of how long-lived cellular immune responses can be induced in vivo after vaccination.

\section{Acquired cellular immunity}

The acquired cellular immune response is composed of $\mathrm{CD}^{+}$and $\mathrm{CD}^{+}{ }^{+}$cells. $\mathrm{CD} 4^{+} \mathrm{T}$ cells recognize proteins commonly referred to as antigens - after they have been processed by a specific group of cells termed antigenpresenting cells. $\mathrm{CD} 4^{+} \mathrm{T}$ cells recognize antigens that have been processed through the exogenous pathway by antigen- presenting cells (such as dendritic cells, macrophages and B cells) expressing major histocompatibility complex (MHC) class II molecules (Fig. 1). After the recognition of antigen in association with MHC class II molecules, $\mathrm{CD}^{+}{ }^{+} \mathrm{T}$ cells become activated, and differentiation can occur into functional subsets termed T helper $1\left(\mathrm{~T}_{\mathrm{H}} 1\right)$-type and T helper 2 $\left(\mathrm{T}_{\mathrm{H}} 2\right)$-type cells. The distinction of these subsets is based on their production of a variety of proteins called cytokines. The signature cytokines for $\mathrm{T}_{\mathrm{H}} 1$ and $\mathrm{T}_{\mathrm{H}} 2$ cells are interferon (IFN) $-\gamma$ and interleukin (IL)-4, respectively. $\mathrm{T}_{\mathrm{H}} 1$ cells, through their production of IFN- $\gamma$, mediate the killing of organisms responsible for a variety of intracellular infections. For many intracellular infections, the induction of a functional $\mathrm{T}_{\mathrm{H}} 1$ response is crucially dependent on another cytokine, IL-12, which is produced by antigen-presenting cells such as macrophages or dendritic cells after exposure to the pathogen at the initiation of the immune response. Thus, in response to many intracellular infections, IL-12 is the inducer cytokine of $\mathrm{T}_{\mathrm{H}} 1$ cells, and IFN- $\gamma$ is the effector cytokine that mediates protection. $\mathrm{CD}^{+} \mathrm{T}$ cells recognize antigens that are processed and presented by cells expressing MHC class I molecules. $\mathrm{CD}^{+} \mathrm{T}$ cells also mediate their effector function through the production of cytokines such as IFN- $\gamma$ and tumour necrosis factor (TNF)- $\alpha$ and/or through a direct cytolytic mechanism (Fig. 1). The mechanism of cytolytic killing can be mediated by the release of granule contents such as perforin and granzyme from $\mathrm{CD} 8^{+}$ $\mathrm{T}$ cells. In addition, $\mathrm{CD} 8^{+} \mathrm{T}$ cells can kill cells by a process of Fas-mediated lysis.

The role of $T_{H} 1$ and $C D 8^{+} T$ cells in protective immunity

In many experimental rodent models of intracellular infection, which include malaria and Mycobacterium tuberculosis, mice made deficient in genes for IFN- $\gamma$ or IL-12 or functionally depleted of $\mathrm{CD} 8^{+} \mathrm{T}$ cells have all been shown to have an increased susceptibility to infection, with accelerated mortality. Moreover, humans with specific mutations in their receptors for IFN- $\gamma$ or IL-12 have been shown to have enhanced susceptibility to mycobacterial disease. Together, these results provide strong evidence that for tuberculosis, malaria and other parasitic infections including Leishmania major, $\mathrm{T}_{\mathrm{H}} 1$ and/or $\mathrm{CD} 8^{+} \mathrm{T}$-cell responses are the effector mechanisms required for protective immunity. 
Finally, there is evidence that $\mathrm{CD} 8^{+} \mathrm{T}$ cells also have a crucial role in mediating protection against HIV infection in both primates and humans. Thus, the development of a successful vaccine for the these diseases will be facilitated by a thorough understanding of how cellular immune responses are generated and maintained in vivo.

\section{Cellular memory}

The major hallmarks of the immune response are discrimination between self and non-self, and immune memory. Whether it relates to cellular or humoral immunity, immune memory is the conceptual basis for how vaccines work. With regard to cellular memory, $\mathrm{CD} 4^{+}$ and $\mathrm{CD}^{+}$memory $\mathrm{T}$ cells differ from naive $\mathrm{T}$ cells in the following manner. First, memory cells express specific cell-surface markers that can denote prior activation. Second, memory cells are qualitatively different from naive $\mathrm{T}$ cells and seem to have less stringent requirements for activation. In this regard, memory cells respond to a previously encountered antigen or pathogen (secondary response) more vigorously and faster ${ }^{1,2}$ than naive T cells. Furthermore, memory cells can be further divided into 'resting' and 'effector' cells. Effector memory cells are usually large, whereas resting memory cells are small. Effector $\mathrm{CD}^{+} \mathrm{T}$ cells $\left(\mathrm{T}_{\mathrm{H}} 1\right.$ cells) readily secrete cytokines, whereas effector $\mathrm{CD} 8^{+} \mathrm{T}$ cells have direct cytolytic activity ex vivo. By contrast, resting memory $\mathrm{CD} 4^{+}$or $\mathrm{CD}^{+} \mathrm{T}$ cells need to be stimulated with antigen before producing cytokines or acquiring cytolytic activity, respectively. Finally, a recent study ${ }^{3}$ suggests that differential expression of the chemokine receptor CCR7 segregates human memory $\mathrm{T}$ cells into two functionally distinct subsets. CCR7 ${ }^{+}$cells have been referred to as 'central' memory cells and express lymph-node homing receptors but lack immediate effector function. CCR7 ${ }^{-}$cells display immediate effector function and have been referred to as 'effector' memory cells. Thus, the differential expression of CCR7 on T cells permits the existence of two separate pools of memory cells that are dynamic and can serve to mediate effector function in response to infection at different sites. It should also be noted that, after activation, $\mathrm{CCR} 7^{+}$cells differentiate into CCR7 ${ }^{-}$cells and acquire effector function. These results support a linear differentiation model in which naive $\mathrm{T}$ cells first develop into central memory $\left(\mathrm{CCR} 7^{+}\right)$and then proceed to effector memory cells $\left(\mathrm{CCR} 7^{-}\right)^{3}$. Two crucial factors that are most likely to regulate the generation, maintenance and effector aspects of T-cell memory responses and the transition from $\mathrm{CCR}^{+}$to $\mathrm{CCR} 7^{-}$cells are antigen and cytokines.

Requirement for antigen in maintaining cellular memory

In recent years there has been tremendous interest in whether antigen is required to sustain memory $\mathrm{T}$ cells. This issue has been controversial in that some reports show that antigen is required to maintain memory cells ${ }^{4-6}$, whereas others find that memory $\mathrm{CD} 8^{+} \mathrm{T}$ cells can be maintained in an antigen-independent manner ${ }^{7-9}$. It is currently our view, on the basis of all the evidence, that antigen is not essential to sustain memory cells in vivo. Moreover, in terms of cytokine production (namely IFN- $\gamma$ ) for both $\mathrm{CD} 4^{+}$and $\mathrm{CD} 8^{+} \mathrm{T}$ cells ${ }^{8,9}$, rapid responsiveness can also be maintained from long-lived memory $\mathrm{T}$ cells in the absence of antigen. But as it relates to immune protection in diseases in which $\mathrm{CD}^{+} \mathrm{T}$ cells are important (such as malaria and HIV) there is strong evidence that antigen might be required to mediate a protective response. This could relate to the factors discussed below and is supported by the following results. First, in the rodent model of malaria infection after inoculation, parasites quickly enter the liver and infect hepatocytes. There is a marked expansion in parasite growth over the next several days, and then parasites exit the liver and enter the circulation, where they infect red blood cells. Because parasites infect hepatocytes, which express MHC class I, they are targets for $\mathrm{CD}^{+} \mathrm{T}$-cell cytolytic activity. In this regard in a rodent model of malaria infection, after vaccination with DNA encoding specific malarial antigens, protection was induced in a manner dependent on $\mathrm{CD}^{+} \mathrm{T}$ cells and IFN- $\gamma$ (ref. 10). These and other results clearly establish the importance of $\mathrm{CD}^{+} \mathrm{T}$ cells in protection against malaria in this rodent model. In addition, vaccination with

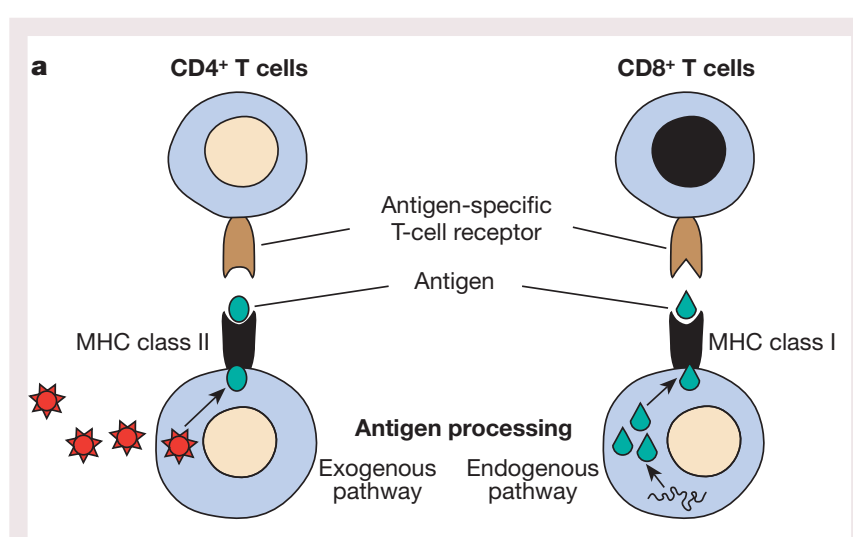

b
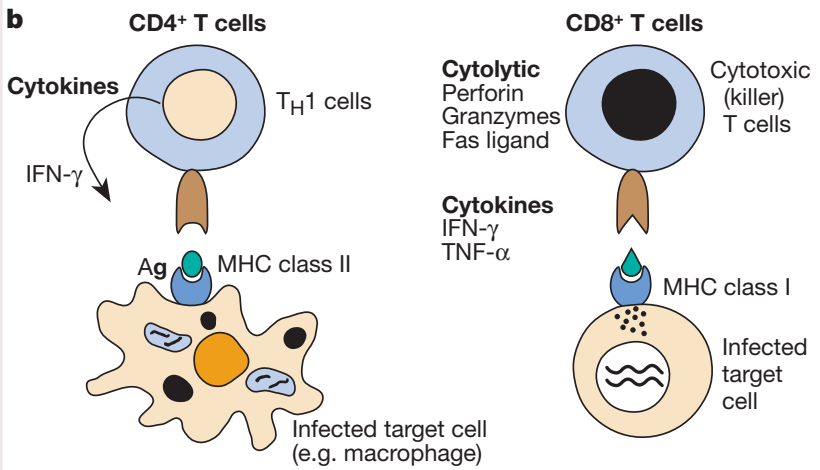

Figure 1 Mechanism of T-cell activation and effector function. a, Mechanism of antigen processing and recognition by $T$ cells. $\mathbf{b}$, Effector function of $T_{H} 1$ and $C D 8^{+} T$ cells. Ag, antigen.

live irradiated sporozoites confers protection in rodents, primates and humans against a challenge with live unirradiated sporozoites ${ }^{11}$. Protective immunity after vaccination with irradiated sporozoites in rats was abrogated by treatment with a drug that eliminated the pre-existing parasites in the liver before re-challenge ${ }^{12}$. Thus, although the immune mechanism by which irradiated sporozoites are mediating their protective effect is not clear at present, these data suggest that persistent antigen might be required to maintain protection. Moreover, one could speculate that in sporozoite-vaccinated mice, persistent antigen (that is, sporozoites) was required to sustain $\mathrm{CD}^{+} \mathrm{T}$-cell responses that were mediating protection. In infection with HIV, there is also strong correlative evidence that enhanced $\mathrm{CD}^{+} \mathrm{T}$ cell cytolytic activity is associated with a reduced viral load in infected primates ${ }^{13,14}$ and in patients with $\mathrm{HIV}^{15}$. Moreover, by monitoring the frequency of HIV-specific $\mathrm{CD} 8^{+} \mathrm{T}$ cells using MHC class I-specific tetramers, it was shown that treatment of patients with highly active antiretroviral therapy (HAART) caused a decrease in the number of antigen-specific $\mathrm{CD}^{+}$cells $^{15,16}$. This potential ability of antigen (viral load) to sustain $\mathrm{CD}^{+}$cytolytic activity is the basis for current clinical trials in which HIV-infected patients on HAART have structured interruptions of their treatment to expose the immune system briefly to antigen and thus sustain the cytolytic response ${ }^{16,17}$. It is hoped that this strategy will generate a persistent cellular immune response that can serve to limit viral replication even after therapy ceases.

With regard to $C D 4^{+} \mathrm{T}$ cell memory ( $\mathrm{T}_{\mathrm{H}} 1$ memory), it has been demonstrated that after the adoptive transfer of short-term $\mathrm{T}_{\mathrm{H}} 1$ lines that were generated in vitro, cells are maintained in vivo for several months in the absence of antigen or even MHC class II molecules ${ }^{18}$. Thus, from these results, it is clear that antigen is not required for sustaining a pool of 'resting' memory $\mathrm{T}_{\mathrm{H}} 1$ cells that were generated in 
vitro. Moreover, in a different adoptive transfer model, it was also demonstrated that CD4 memory T cells could be sustained in the absence of antigen ${ }^{19}$. In this study, it was of interest that not all memory $\mathrm{T}$ cells expressed IFN- $\gamma$ when assessed ex vivo. These results suggested that there could be a heterogeneous population of memory $\mathrm{CD}^{+} \mathrm{T}$ cells. One type of memory cell could rapidly produce IFN- $\gamma$ after stimulation, whereas the other type of memory cell required additional differentiation for effector function. These results might be analogous to the delineation of effector/memory cells $\left(\right.$ CCR7 ${ }^{-}$) and central/memory cells $\left(\mathrm{CCR}^{+}\right)$, respectively, as discussed above. What has not been well defined is whether persistent antigen is required for the maintenance of protective levels of memory/effector $\mathrm{T}_{\mathrm{H}} 1$ cells that are generated in vivo after immunization. Some insight into the importance of antigen in maintaining immunity can be gained from observations in the course of human clinical disease. As noted above, $\mathrm{T}_{\mathrm{H}} 1$ cells have a clear role in mediating protection in rodent models of M. tuberculosis and cutaneous infection with Leishmania. In most people with tuberculosis or cutaneous infection with Leishmania, once infected individuals have resolved their initial infection they remain resistant to reinfection. Moreover, reactivation of tuberculosis can occur long after the resolution of primary infection in immunocompromised subjects, suggesting the existence of persistent live mycobacteria. In addition, persistent antigen has been documented in cutaneous infection with Leishmania many years after primary infection ${ }^{20}$. Taken together, these results suggest that persistent antigen might be important in sustaining immunity in diseases that require $\mathrm{T}_{\mathrm{H}} 1$ cells and/or $\mathrm{CD} 8^{+}$cytolytic cells. Finally, although persistent antigen might be important for long-term immunity that is induced by primary infection or by vaccination for the diseases discussed here, there is also substantial evidence indicating that too much antigen can markedly impair T-cell immune responses. This could occur quantitatively by deletion of activated cells through antigen-induced cellular death (apoptosis) and/or qualitatively by inducing unresponsiveness of the $\mathrm{T}$ cell (clonal anergy). Thus, although the persistence of antigen might be important in conferring immune protection after vaccination, the amount of antigen present might also be a crucial factor in optimizing longterm T-cell immunity.

Requirement for cytokines in maintaining cellular memory

Another factor besides antigen that is crucial for generating and sustaining memory $\mathrm{T}$ cells is the role of cytokines. For $\mathrm{CD} 8^{+} \mathrm{T}$ cells, there is evidence that IFN- $\alpha$ acting through IL-15 might be important in sustaining memory $\mathrm{CD}^{+} \mathrm{T}$-cell responses ${ }^{21}$. As noted above, $\mathrm{CD} 4^{+} \mathrm{T}$ cells require IL-12 for differentiation into functional $\mathrm{T}_{\mathrm{H}} 1$ cells. Moreover, there is now evidence that IL-12 might also be required to sustain memory/effector $\mathrm{T}_{\mathrm{H}} 1$ cells sufficient to mediate a biological outcome. This was shown in a mouse vaccine model of infection with L. major in which it was demonstrated that continuous IL-12 was required to sustain $T_{H} 1$ cells sufficient to control infection after challenge ${ }^{22}$. Finally, $\mathrm{T}_{\mathrm{H}} 1$ responses have also been shown to mediate protection against $M$. tuberculosis. Currently, the live attenuated Bacille Calmette-Guérin (BCG) strain, which is an inducer of IL-12 and $\mathrm{T}_{\mathrm{H}} 1$ responses, does provide some protection against systemic disease early in childhood. By contrast, the efficacy of BCG vaccination against pulmonary disease occurring later in life is quite variable. Although there are many reasons why BCG might not be uniformly effective against pulmonary disease, one potential explanation is that, over time, there is a sterilization of BCG. This would limit the ability both to provide a source of antigen and to induce cytokines such as IL-12.

Thus, for both $\mathrm{T}_{\mathrm{H}} 1$ and $\mathrm{CD} 8^{+} \mathrm{T}$-cell memory responses, the persistence of antigen might not be required for the maintenance of 'resting' memory $\mathrm{CD}^{+}{ }^{+}$or $\mathrm{CD}^{+} \mathrm{T}$ cells but might be crucial for sustaining a pool of 'effectors' sufficient to provide protection for those diseases that require cellular immunity. Moreover, cytokines (such as IL-12) might also be required for the maintenance of a pool of memory/effector $\mathrm{T}_{\mathrm{H}} 1$ cells sufficient for protection. The exact
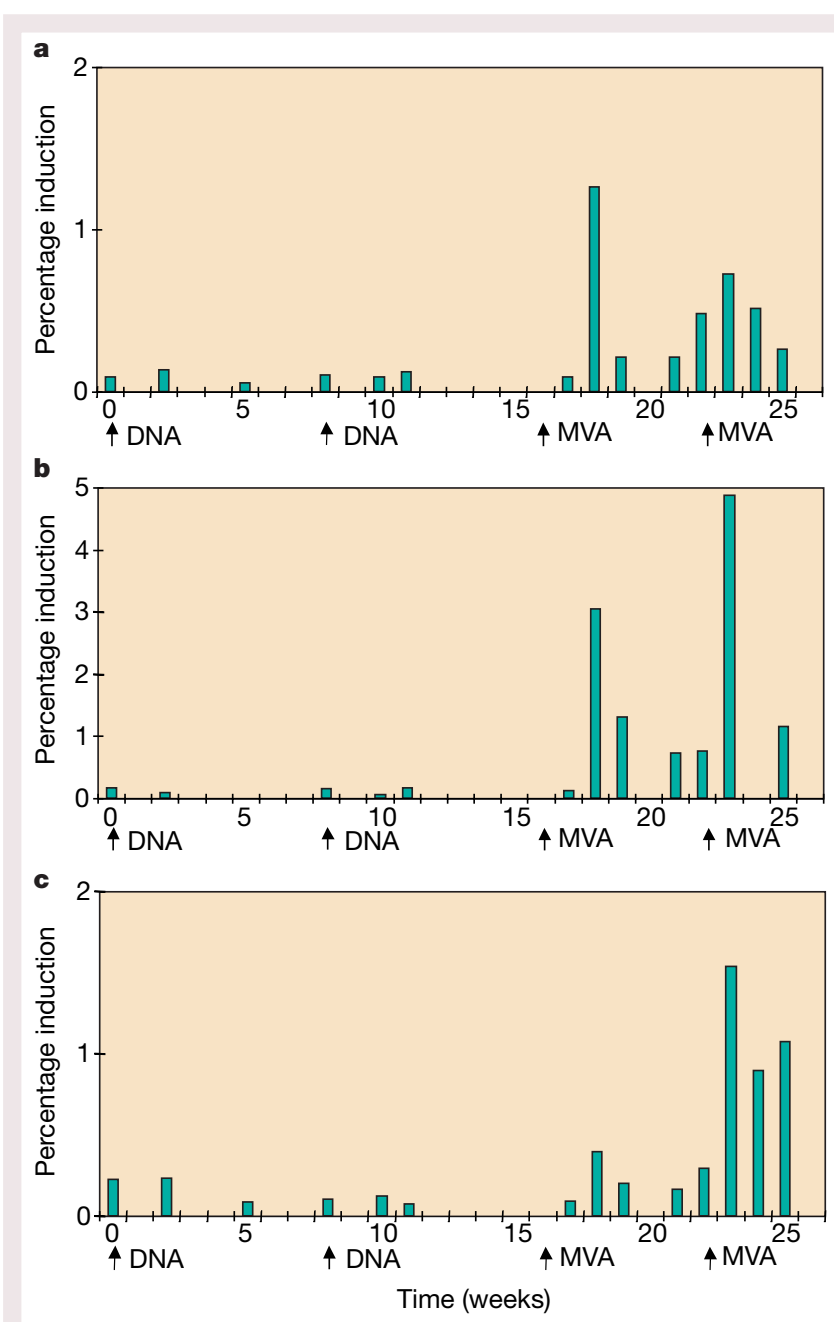

Figure 2 Induction of $\mathrm{CD} 8^{+} \mathrm{T}$ cells in three macaque monkeys $(\mathbf{a}-\mathbf{c})$ with the use of a heterologous prime-boost immunization regime (modified from ref. 46). Macaques were immunized with a plasmid DNA vaccine encoding a SIV CD8 T-cell epitope restricted by the MHC molecule Mamu- $A^{*} 01$ at weeks 0 and 8 , and then immunized with a recombinant modified vaccinia Ankara (MVA) vaccine encoding the same epitope at weeks 16 and 22. The time course (in weeks) of induction of CD8 T cells specific for that epitope was measured by using an MHC-peptide tetramer; induction is expressed as the percentage of total peripheral blood $\mathrm{CD}^{+} T$ cells. Prime-boosted animals showed higher responses than macaques immunized twice with MVA alone ${ }^{46}$.

nature of these requirements depends on additional factors highlighted below.

\section{Immune protection - thresholds, time and place Thresholds}

Most current vaccines require multiple immunizations for the induction of sufficient antibody to mediate protective immunity. In animal models of diseases requiring cellular immunity, there also seems to be a certain threshold of effector $\mathrm{T}_{\mathrm{H}} 1$ or $\mathrm{CD} 8^{+} \mathrm{T}$ cells that is required for protection. For example, in rodent malaria a threshold level of about 400 IFN- $\gamma$-secreting peptide-specific $C D 8^{+} \mathrm{T}$ cells per million splenocytes is required to protect against sporozoite challenge $e^{23}$. In addition to the magnitude or threshold of the cellular response, it is also important to understand the ontogeny of the immune response after immunization or infection. In this regard, the induction of antigen-specific $\mathrm{CD}^{+}$and $\mathrm{CD} 8^{+} \mathrm{T}$ cells in vivo proceeds through three phases. First, after immunization or exposure to natural infection, there is an initial expansion phase in which there is a substantial increase in the frequency of antigen-specific cells ${ }^{24,25}$. 


\begin{tabular}{|c|c|c|c|c|c|}
\hline & & DNA vaccine & Live attenuated & Killed protein subunit & Live vector (e.g. poxvirus) \\
\hline \multicolumn{6}{|l|}{ Immune response } \\
\hline Humoral & B cells & ++ & +++ & +++ & ++ \\
\hline \multirow[t]{2}{*}{ Cellular } & $\mathrm{CD}^{+}$ & $+++T_{H} 1^{*}$ & $\pm T_{H} 1$ & $\pm T_{H} 1$ & + \\
\hline & $\mathrm{CD8}^{+}$ & ++ & +++ & - & +++ \\
\hline Antigen presentation & MHC class & I and II & I and II & $\|$ & I and II \\
\hline \multicolumn{6}{|l|}{ Memory } \\
\hline Cellular & & ++ & +++ & \pm & ++ \\
\hline \multicolumn{6}{|l|}{ Manufacturing } \\
\hline \multicolumn{2}{|l|}{$\begin{array}{l}\text { Ease of development } \\
\text { and production }\end{array}$} & ++++ & + & ++ & +++ \\
\hline \multicolumn{2}{|l|}{ Cost } & +++ & + & + & ++++ \\
\hline \multicolumn{2}{|l|}{ Transport/storage } & $+\cdots$ & + & +++ & + \\
\hline \multicolumn{2}{|l|}{ Safety } & $+++\dagger$ & $++\ddagger$ & ++++ & ++ \\
\hline
\end{tabular}

${ }^{*} T_{H} 2$ responses can be induced by gene-gun immunization in mice.

†Data available only from phase I trials.

‡Live/attenuated vaccines may be precluded for use in immunocompromised patients and certain infections such as HIV.

This expansion phase, which usually occurs during the first week, is followed by the death phase, in which more than $90 \%$ of the activated cells undergo activation-induced apoptosis ${ }^{26}$. For $\mathrm{CD}^{+} \mathrm{T}$ cells, the initial frequency of antigen-specific cells seems to be proportional to the antigenic load. In general, live infection induces a greater increase in the frequency of antigen-specific cells than immunizations with specific peptide, DNA vaccines or recombinant non-replicating viruses encoding the specific antigen ${ }^{24}$. Moreover, it has been suggested that the persistence of antigen ${ }^{27}$ and/or the amount of antigen $^{28}$ also affects qualitative aspects of $\mathrm{CD}^{+} \mathrm{T}$-cell memory/effector responses. Once the initial expansion and death phases have occurred, the third phase of the response - the memory phase - can be stably maintained over a long period, even in the absence of persistent antigen ${ }^{1,7,29}$. This memory phase of the response in the presence or absence of persistent antigen is likely to be heterogeneous, consisting of 'central' and 'effector' memory cells. The requirement for antigen in sustaining these specific memory cell types is not clear at present and is likely to have a large effect on whether or not immune protection is achieved, depending on the particular type of infection. Finally, the magnitude of the memory phase is generally determined by the size of the initial clonal burst induced by immunization $^{30}$. Thus, for protective memory $\mathrm{CD}^{+} \mathrm{T}$-cell responses for diseases such as HIV and malaria that might require a high number of memory/effector cells, it is desirable to use a vaccine regimen that provides a sufficient antigenic load to maximize the initial burst size, so that a threshold of memory cells is maintained that will be protective.

Time and place

Currently used vaccines work by either preventing infection or preventing disease. For certain infections such as HIV, which can remain latent for long periods before progressing to clinical infection, the prevention of any infection by a vaccine would be optimal. Prevention of primary infection is probably mediated by a humoral immune response. By contrast, $\mathrm{CD} 8^{+} \mathrm{T}$ cells exert their effector function by recognizing antigen that has been processed in the context of MHC class I, and act to kill infected cells and to prevent a further spread of the infection. Thus, although $\mathrm{CD} 8^{+} \mathrm{T}$ cells might not themselves be able to prevent infection, they still could have a beneficial role in limiting or preventing disease, depending on how efficient they are in killing all of the infected cells. Furthermore, the effectiveness of the cellular immune response depends on where exposure to the pathogen occurs, and on whether the immune response exists within that site or needs to migrate from an immune organ. This crucial factor of time and place is determined by the specific infection. Thus, for rapidly replicating viruses such as influenza, it has been shown that the ability to generate long-term memory $\mathrm{CD}^{+} \mathrm{T}$ cells after primary infection is not sufficient to prevent disease in the lungs after re-challenge ${ }^{31}$. In this study, even though the frequency and kinetics of the secondary $\mathrm{CD} 8^{+} \mathrm{T}$-cell responses in the lungs were enhanced, it still took several days for cells to localize to the lungs from the mediastinal lymph nodes. With regard to HIV infection, it has recently been shown that after the intravaginal inoculation of rhesus monkeys with simian immunodeficiency virus (SIV), it was possible to find $\mathrm{CD}^{+} \mathrm{T}$ cells in the cervix that contained viral RNA by 3 days after infection ${ }^{32}$. These results imply a relatively narrow time window during which effector $\mathrm{CD} 8^{+} \mathrm{T}$ cells need either to be present at the mucosal site or to migrate quickly from a systemic site. At present, generating a high frequency of effector $\mathrm{CD}^{+}$cytolytic responses at mucosal sites has been difficult and represents a daunting challenge for vaccines. Nevertheless, the ability to generate a pool of 'systemic' memory $\mathrm{CD}^{+} \mathrm{T}$ cells that can be activated after exposure to HIV might not prevent infection or kill all infected cells, but could still limit the initial viral set point after acute infection, providing some measure of protection ${ }^{33}$. In contrast with certain viral infections in which infection, latency and clinical disease can be established relatively quickly, time might be less of a limiting factor for protection against certain parasitic or mycobacterial infections. For malaria, the pre-erythrocytic liver stage of infection lasts for about 7 days in humans, providing a potentially long window for the action of effector T cells. It is important to note that complete killing of all parasites must occur within the liver to prevent release into the circulation and the infection of red blood cells. Finally, for pulmonary tuberculosis or cutaneous leishmanial infection, the time between initial exposure and clinical disease can be several weeks or longer. In these infections, it might be possible for a low frequency of 'resting' memory T cells that are generated after vaccination to develop into effector $\mathrm{T}$ cells that can control and prevent clinical disease. The challenges that lie ahead for vaccine development will be not only to optimize the qualitative and quantitative cellular immune response but also to induce responses directly at sites of infection.

Vaccines inducing cellular immunity to intracellular pathogens All currently licensed vaccines (killed or inactivated, whole-cell, recombinant protein, or live attenuated) generate humoral immune responses. A potential shortcoming of non-live vaccines is their relative inefficiency in generating cellular immune responses $\left(\mathrm{T}_{\mathrm{H}} 1\right.$ or $\mathrm{CD}^{+} \mathrm{T}$-cell responses), thus limiting their application for those diseases requiring cellular immunity (Table 1). Moreover, although certain adjuvants (used to enhance the potency of the vaccine) given with non-live vaccines have been shown to induce $\mathrm{T}_{\mathrm{H}} 1$ responses, it has been difficult to find any adjuvant that can facilitate $\mathrm{CD} 8^{+} \mathrm{T}$-cell induction in humans. In contrast, live attenuated vaccines permit the efficient MHC class I presentation of antigen that can stimulate $\mathrm{CD} 8^{+}$ $\mathrm{T}$-cell responses. The ability of live attenuated vaccines to induce 
$\mathrm{T}_{\mathrm{H}} 1$-specific responses in humans, however, is less well characterized and can depend on the specific pathogen. Live vaccines also have the advantage of antigen persistence. From a practical standpoint, and also from one of safety, attenuated whole-organism vaccines raise several issues that can preclude their widespread application for certain diseases (namely HIV and malaria). Thus, the demonstration that DNA vaccines could induce both humoral and broad cellular responses (cytolytic and $\mathrm{T}_{\mathrm{H}} 1$ responses) ${ }^{34}$ in small animals provided hope that they could become the future vaccine of choice for infections requiring cellular immunity in humans ${ }^{35}$. This optimism must be tempered by the fact that the induction of humoral and cellular immune responses after vaccination of humans with DNA has been disappointing in comparison with the rodent model $s^{36-38}$. This has prompted investigators to try to optimize the immunogenicity of plasmid DNA vaccines themselves and/or to use DNA vaccines in combination with other vaccines to enhance immunity.

In terms of optimizing DNA vaccines in humans, several approaches are being taken to improve immunogenicity. In this regard, one approach has focused on specific nucleotide sequences (CpG motifs) within the plasmid. In both mice and humans, CpG oligodeoxynucleotides have been shown to stimulate multiple types of immune cell, leading to enhanced $\mathrm{T}_{\mathrm{H}} 1$ and cytolytic $\mathrm{CD}^{+} \mathrm{T}$-cell responses $^{39-42}$. Moreover, recent work has shown that the immunostimulatory activity of $\mathrm{CpG}$ oligodeoxynucleotides can be blocked by certain non-CpG motifs ${ }^{43}$. Thus, there is currently an effort to determine whether the addition of specific stimulatory CpG motifs, and the deletion of inhibitory ones, within the plasmid backbone can improve their immunogenicity. With regard to combining DNA with other types of vaccine, there have been several studies evaluating prime-boost strategies with vaccines based on live virus on or protein. Live virus vectors themselves typically generate stronger cellular immune responses than do DNA vaccines in small animals, yet clinical trials of poxvirus and adenovirus vectors have generally yielded fairly small cellular immune responses. The enhanced immunogenicity of a heterologous prime-boost immunization strategy, by using DNA priming and boosting with a recombinant poxvirus vector that encodes the same foreign antigens, has been demonstrated in several infectious disease ${ }^{23,44}$. In mice and in primates (Fig. 2), substantial enhancement of $\mathrm{CD} 8^{+} \mathrm{T}$-cell responses was observed, and the immunization order was crucial for maximal immunogenicity. Several poxviruses, such as modified vaccinia virus Ankara (MVA) and fowlpox, and also replication-defective adenoviruses, have this capacity to boost a primed $\mathrm{CD} 8^{+} \mathrm{T}$-cell response substantially ${ }^{45,46}$. Clinical trials of this approach for malaria with MVA as a boosting agent are in progress. Finally, although the current approach of using a DNA prime and a MVA boost is clearly important in optimizing the magnitude of the $\mathrm{CD}^{+}$response, it is not clear how long the enhanced responses will be maintained after the MVA boost. But this raises the issue of whether continued boosting will be required for sustaining a sufficient number of cells to confer protection.

Another method for optimizing the qualitative and quantitative immune response after vaccination is to use an adjuvant. Currently, the only widely used adjuvant in humans is alum, which improves antibody production but has little effect on $\mathrm{T}_{\mathrm{H}} 1$ or $\mathrm{CD}^{+} \mathrm{T}$-cell responses. A novel adjuvant consisting of QS21, monophosphoryl lipid A (MPL) and an oil-in-water emulsion, SBAS2, has been shown to generate both strong antibody responses and $\mathrm{T}_{\mathrm{H}} 1$-type responses in combination with a protein antigen in malaria. This ' $\mathrm{RTS}, \mathrm{S}$ ' vaccine has shown impressive but relatively short-lived protective efficacy in sporozoite challenge studies and is currently undergoing field trials ${ }^{47}$.

\section{Perspective}

Various new vaccination strategies for inducing strong cellular immune responses are now reaching clinical trials. Several of these are being evaluated for diseases such as malaria or HIV or in a therapeutic setting where efficacy can be assessed in small studies. In addition, as noted above, other immunization approaches will probably be required for inducing effective mucosal immunity and for generating different types of T-cell response such as CD1-and other non-classically restricted T cells. Although not all of these new approaches will generate sufficiently durable immunity for useful immunoprophylaxis, even the short-term potent induction of $\mathrm{T}$ effector cells might be of value for therapeutic vaccination. Furthermore, even if vaccines fail to induce long-term cellular immune responses, if exposure occurs within a short time of vaccination - a period during which the immune response is robust - protection could be achieved, and then life-long immunity would be possible as a result of the boosting effect of the live infection. The progress in developing vaccines for cellular immunity will also be enhanced by recent advances in the monitoring of T-cell responses by using sensitive enzyme-linked immunospot techniques and new tetrameric reagents. These tools provide excellent opportunities for the identification of immunological correlates of protection after vaccination. As a whole, our increased understanding of the immune correlates of protection as well as of the requirements for generating and sustaining cellular immune responses provides a framework for successful vaccine development against those diseases that require cellular immunity.

1. Ahmed, R. \& Gray, D. Immunological memory and protective immunity: understanding their relation. Science 272, 54-60 (1996).

2. Dutton, R. W., Bradley, L. M. \& Swain, S. L. T cell memory. Annu. Rev. Immunol. 16, 201-223 (1998).

3. Sallusto, F., Lenig, D., Forster, R., Lipp, M. \& Lanzavecchia, A. Two subsets of memory T lymphocytes with distinct homing potentials and effector functions. Nature 401, 708-712 (1999).

4. Gray, D. \& Matzinger, P. T cell memory is short-lived in the absence of antigen. J. Exp. Med. 174, 969-974 (1991).

5. Bachmann, M. F., Kundig, T. M., Hengartner, H. \& Zinkernagel, R. M. Protection against immunopathological consequences of a viral infection by activated but not resting cytotoxic $\mathrm{T}$ cells: $\mathrm{T}$ cell memory without 'memory T cells'? Proc. Natl Acad. Sci. USA 94, 640-645 (1997).

6. Kundig, T. M. et al. On the role of antigen in maintaining cytotoxic T-cell memory. Proc. Natl Acad. Sci. USA 93, 9716-9723 (1996).

7. Lau, L. L., Jamieson, B. D., Somasundaram, T. \& Ahmed, R. Cytotoxic T-cell memory without antigen. Nature 369, 648-652 (1994).

8. Tanchot, C., Lemonnier, F. A., Perarnau, B., Breitas, A. A. \& Rocha, B. Differential requirements for survival and proliferation of CD8 naïve or memory T cells. Science 276, 2057-2062 (1997).

9. Murali-Krishna, K. et al. Persistence of memory CD8 T cells in MHC class I-deficient mice. Science 286, 1377-1381 (1999).

10. Doolan, D. L. et al. Circumventing genetic restriction of protection against malaria with multigene DNA immunization: $\mathrm{CD}^{+}$cell-, interferon $\gamma$-, and nitric oxide-dependent immunity. J. Exp. Med. 183, 1739-1746 (1996).

11. Guebre-Xabier, M., Schwenk, R. \& Krzych, U. Memory phenotype $\mathrm{CD}^{+} \mathrm{T}$ cells persist in livers of mice protected against malaria by immunization with attenuated Plasmodium berghei sporozoites. Eur. J. Immunol. 29, 3978-3986 (1999).

12. Scheller, L. F. \& Azad, A. F. Maintenance of protective immunity against malaria by persistent hepatic parasites derived from irradiated sporozoites. Proc. Natl Acad. Sci. USA 92, 4066-4068 (1995).

13. Matano, T. et al. Administration of an anti-CD8 monoclonal antibody interferes with the clearance of chimeric simian/human immunodeficiency virus during primary infections of rhesus macaques. J. Virol. 72, 164-169 (1998).

14. Schmitz, J. E. et al. Control of viremia in simian immunodeficiency virus infection by $\mathrm{CD}^{+}$ lymphocytes. Science 283, 857-860 (1999).

15. Ogg, G. S. et al. Quantitation of HIV-1-specific cytotoxic T lymphocytes and plasma load of viral RNA. Science 279, 2103-2106 (1998).

16. Kalams, S. A. et al. Levels of human immunodeficiency virus type 1-specific cytotoxic T-lymphocyte effector and memory responses decline after suppression of viremia with highly active antiretroviral therapy. J. Virol. 73, 6721-6728 (1999).

17. Ortiz, G. M. et al. HIV-1-specific immune responses in subjects who temporarily contain virus replication after discontinuation of highly active antiretroviral therapy. J. Clin. Invest. 104, R13-R18 (1999).

18. Swain, S. L., Hu, H. \& Huston, G. Class II-independent generation of CD4 memory T cells from effectors. Science 286, 1381-1383 (1999).

19. Garcia, S., DiSanto, J. \& Stockinger, B. Following the development of a CD4 T cell response in vivo: from activation to memory formation. Immunity 11, 163-171 (1999).

20. Schuback, A. et al. Detection of Leishmania DNA by polymerase chain reaction in scars of treated human patients. J. Infect. Dis. 178, 911-914 (1998).

21.Zhang, X., Sun, S., Hwang, I., Tough, D. F. \& Sprent, J. Potent and selective stimulation of memoryphenotype $\mathrm{CD}^{+} \mathrm{T}$ cells in vivo by IL-15. Immunity 8, 591-599 (1998).

22. Gurunathan, S., Prussin, C., Sacks, D. L. \& Seder, R. A. Vaccine requirements for sustained cellular immunity to an intracellular parasitic infection. Nature Med. 4, 1409-1415 (1998).

23. Schneider, J. et al. Enhanced immunogenicity for $\mathrm{CD}^{+} \mathrm{T}$ cell induction and complete protective efficacy of malaria DNA vaccination by boosting with modified vaccinia virus Ankara. Nature Med. 4 , 397-402 (1998).

24. Oehen, S., Waldner, H., Kundig, T. M., Hengartner, H. \& Zinkernagel, R. M. Antivirally protective cytotoxic $\mathrm{T}$ cell memory to lymphocytic choriomeningitis virus is governed by persisting antigen. $J$. Exp. Med. 176, 1273-1281 (1992). 


\section{insight progress}

25. McHeyzer-Williams, M. G. \& Davis, M. M. Antigen-specific development of primary and memory T cells in vivo. Science 268, 106-111 (1995).

26. Razvi, E. S. \& Welsh, R. M. Apoptosis in viral infections. Adv. Virus Res. 45, 1-60 (1995).

27. Ochsenbein, A. F. et al. A comparison of T cell memory against the same antigen induced by virus versus intracellular bacteria. Proc. Natl Acad. Sci. USA 96, $9293-9298$ (1999).

28. Opferman, J. T., Ober, B. T. \& Ashton-Rickardt, P. G. Linear differentiation of cytotoxic effectors into memory T lymphocytes. Science 283, 1745-1748 (1999).

29. Mullbacher, A. The long-term maintenance of cytotoxic $\mathrm{T}$ cell memory does not require persistence of antigen. J. Exp. Med. 179, 317-321 (1994).

30. Hou, S., Hyland, L., Ryan, K. W., Portner, A. \& Doherty, P. C. Virus-specific CD8 ${ }^{+}$T-cell memory determined by clonal burst size. Nature 369, 652-654 (1994).

31. Flynn, K. J. et al. Virus-specific $\mathrm{CD} 8^{+} \mathrm{T}$ cells in primary and secondary influenza pneumonia. Immunity 8, 683-691 (1998).

32. Zhang, Z. et al. Sexual transmission and propagation of SIV and HIV in resting and activated $\mathrm{CD} 4^{+} \mathrm{T}$ cells. Science 286, 1353-1357 (1999).

33. Lifson, J. D. et al. The extent of early viral replication is a critical determinant of the natural history of simian immunodeficiency virus infection. J. Virol. 71, 9508-9514 (1997).

34. Ulmer, J. B. et al. Heterologous protection against influenza by injection of DNA encoding a viral protein. Science 259, 1745-1749 (1993)

35. Seder, R. A. \& Gurunathan, S. DNA vaccines - designer vaccines for the 21st century. N. Engl. J. Med. 341, 277-278 (1999).

36. Wang, R. et al. Induction of antigen-specific cytotoxic T lymphocytes in humans by a malaria DNA vaccine. Science 282, 476-480 (1998).

37. Le, T. P. et al. Safety, tolerability and humoral immune responses after intramuscular administration of a malaria DNA vaccine to healthy adult volunteers. Vaccine 18, 1893-1901 (2000),
38. Calarota, S. et al. Cellular cytotoxic response induced by DNA vaccination in HIV-1-infected patients. Lancet 351, 1320-1325 (1998).

39. Cho, H. J. et al. Immunostimulatory DNA-based vaccines induce cytotoxic lymphocyte activity by a T-helper cell-independent mechanism. Nature Biotechnol. 18, 509-514 (2000).

40. Cowdery, J. S., Chace, J. H., Yi, A. K. \& Krieg, A. M. Bacterial DNA induces NK cells to produce IFN- $\gamma$ in vivo and increases the toxicity of lipopolysaccharides. J. Immunol. 156, 4570-4575 (1996).

41. Klinman, D. M., Yi, A. K., Beaucage, S. L., Conover, J. \& Krieg, A. M. CpG motifs present in bacterial DNA rapidly induce lymphocytes to secrete interleukin 6, interleukin 12, and interferon $\gamma$. Proc. Natl Acad. Sci. USA 93, 2879-2883 (1996).

42. Sato, Y. et al. Immunostimulatory DNA sequences necessary for effective intradermal gene immunization. Science 273, 352-354 (1996).

43. Krieg, A. M. et al. Sequence motifs in adenoviral DNA block immune activation by stimulatory $\mathrm{CpG}$ motifs. Proc. Natl Acad. Sci. USA 95, 12631-12636 (1998).

44. Schneider, J. et al. Induction of $\mathrm{CD}^{+} \mathrm{T}$ cells using heterologous prime-boost immunisation strategies. Immunol. Rev. 170, 29-38 (1999).

45. Kent, S. J. et al. Enhanced T-cell immunogenicity and protective efficacy of a human immunodeficiency virus type 1 vaccine regimen consisting of consecutive priming with DNA and boosting with recombinant fowlpox virus. J. Virol. 72, 10180-10188 (1998).

46. Hanke, T. et al. Effective induction of simian immunodeficiency virus-specific cytotoxic T lymphocytes in macaques by using a multiepitope gene and DNA prime-modified vaccinia virus Ankara boost vaccination regimen. J. Virol. 73, 7524-7532 (1999).

47. Stoute, J. A. et al. A preliminary evaluation of a recombinant circumsporozoite protein vaccine against Plasmodium falciparum malaria. N. Engl. J. Med. 336, 86-91 (1997).

Correspondence and requests for materials should be addressed to R.A.S. (e-mail: rseder@niaid.nih.gov). 